\title{
AXIOM OF CHOICE AND COMPLEMENTATION
}

\author{
RADU DIACONESCU
}

ABSTRACT. It is shown that an intuitionistic model of set theory with the axiom of choice has to be a classical one.

A topos $\mathcal{E}$ is a category which has finite limits (i.e. finite products, intersections and a terminal object, 1), a universal monomorphism $1 \stackrel{\text { tue }}{\longleftrightarrow} \Omega$ (i.e. for any monomorphism of $\mathscr{G} A \stackrel{m}{\longrightarrow} A$ there exists a unique "characteristic function" such that the diagram

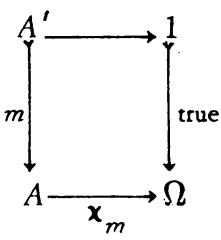

is a pull-back), and for each object its power set $\Omega^{A}$ (this is characterized by the fact that the morphisms $X \rightarrow \Omega^{A}$ are precisely the subobjects of $X \times A$, in particular its global sections $1 \rightarrow \Omega^{A}$ are the subobjects of $A$ ). The most common examples of topos are the category of sets, $\mathcal{S}$, categories of functors $S\left(C^{\mathrm{op}}\right)$ for any small category $\mathrm{C}$, and categories of sheaves on topological spaces. Details about these can be found in [1] or [2]. One of the main consequences of the axioms is that $\Omega$ (the "truth table" object) is a Heyting alyebra object. (A Heyting algebra is a lattice with "pseudocomplements". The open set lattice of a topological space is a typical example.) Roughly speaking a topos could be thought of as a model for intuitionistic set theory (subobjects do not have honest complements).

In this setting the axiom of choice reads:

AC: Every epimorphism has a section.

Theorem. Any coequalizer of two nonintersecting monomorphisms bas a section iff in $\&$ subobjects have complements.

Proof. Let $A^{\prime} \stackrel{m}{\longrightarrow} A$ be a monomorphism in $\mathcal{E}$ and construct the fol-

Received by the editors May 20, 1974 . 18B05.

AMS (MOS) subject classifications (1970). Primary 02K20; Secondary 18A15, 
lowing coequalizer diagram:

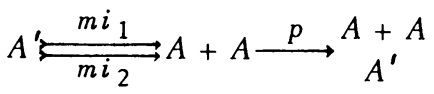

in which by hypothesis $p$ has a splitting. $\begin{gathered}A+A \\ A^{\prime}\end{gathered}$ can also be obtained from the push-out diagram:

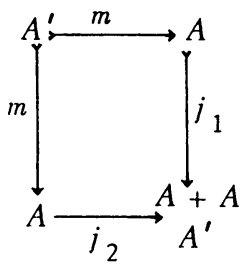

From the general theory of topos it follows that $j_{1}$ and $j_{2}$ are monomorphisms and that (2) is a pull-back (hence an intersection).

Let $A+A \stackrel{s}{\longrightarrow} A+A$ be a section of $p$. The mere existence of such a morphism (see [1] or [2]) forces $A+A$ to be the form $D+E$ where $D=$ $s^{-1}(A+0), E=s^{-1}(0+A)$. Similarly $s \cdot j_{1}$ and $s \cdot j_{2}$ produce two decompositions of $A$, namely $A=B_{1}+C_{1}$ and $A=B_{2}+C_{2}$, hence

$$
A=B_{1} \cap B_{2}+B_{1} \cap C_{2}+C_{1} \cap B_{2}+C_{1} \cap C_{2} \text {. }
$$

Thus the diagram (2) becomes

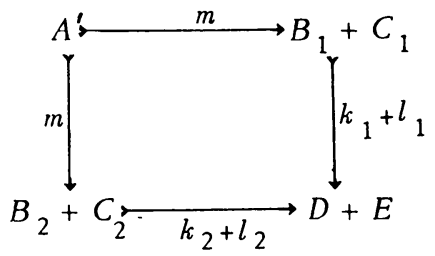

therefore $A^{\prime}=B_{1} \cap B_{2}+C_{1} \cap C_{2}$ and obviously has a complement $\neg A^{\prime}=$ $B_{1} \cap C_{2}+C_{1} \cap B_{2}$

Conversely, if

$$
A^{\prime} \underset{n}{\stackrel{m}{\rightleftarrows}} A
$$

are such that $m \cap n=0$ then $A=m+\neg m$ and $A=n+\neg n$, hence

$$
A=m \cap \neg n+\neg m \cap n+-\mid m \cap \neg n=m+n+\neg m \cap \neg n
$$

i.e. $A=A^{\prime}+A^{\prime}+B$ for some object $B$. But then the coequalizer of $m$ and $n$ is 


$$
A^{\prime} \underset{i_{2}}{\stackrel{i_{1}}{\longleftarrow}} A^{\prime}+A^{\prime}+B \stackrel{\nabla+B}{\longrightarrow} A^{\prime}+B
$$

which is obviously split by

$$
A^{\prime}+B \stackrel{i_{1}+B}{\longrightarrow} A^{\prime}+A^{\prime}+B
$$

Corollary. AC implies that every subobject bas a complement.

Corollary. If in Sh $(T)$ epimorphisms (or even only coequalizers of nonintersecting monomorphisms) split then every open set in $T$ is clopen and $T$ is the disjoint union of sets with the indiscrete topology.

The present version of the proof is the result of several discussions with M. Barr in which he pointed out that the amount of topos language can be reduced to a minimum.

\section{REFERENCES}

1. F. W. Lawvere (editor), Toposes, Algebraic Geometry and Logic, Lecture Notes in Math., vol. 274, Springer-Verlag, Berlin and New York, 1972.

2. M. Tierney, Axiomatic sheaf theory, Some Constructions and Applications in Categories and Commutative Algebra (P. Salmon, editor), Edizioni Cremonese, Roma, 1973. CANADA

DEPARTMENT OF MATHEMATICS, McGILL UNIVERSITY, MONTREAL, QUEBEC, 